\title{
JackHammer: Efficient Rowhammer on Heterogeneous FPGA-CPU Platforms
}

\author{
Zane Weissman ${ }^{1}$, Thore Tiemann ${ }^{2}$, Daniel Moghimi ${ }^{1}$, Evan Custodio $^{3}$, \\ Thomas Eisenbarth ${ }^{2}$ and Berk Sunar ${ }^{1}$ \\ 1 Worcester Polytechnic Institute, MA, USA \\ zweissman@wpi .edu, amoghimi@wpi.edu, sunar@wpi.edu \\ ${ }^{2}$ University of Lübeck, Lübeck, Germany \\ thore.tiemann@student.uni-luebeck.de, thomas.eisenbarth@uni-luebeck.de \\ ${ }^{3}$ Intel Corporation, Hudson, MA, USA \\ evan.custodio@intel.com
}

\begin{abstract}
After years of development, FPGAs are finally making an appearance on multi-tenant cloud servers. Heterogeneous FPGA-CPU microarchitectures require reassessment of common assumptions about isolation and security boundaries, as they introduce new attack vectors and vulnerabilities. In this work, we analyze the memory and cache subsystem and study Rowhammer and cache attacks enabled by two proposed heterogeneous FPGA-CPU platforms from Intel: the Arria $10 \mathrm{GX}$ with an integrated FPGA-CPU platform, and the Arria 10 GX PAC expansion card which connects the FPGA to the CPU via the PCIe interface. We demonstrate JackHammer, a novel, efficient, and stealthy Rowhammer from the FPGA to the host's main memory. Our results indicate that a malicious FPGA can perform twice as fast as a typical Rowhammer from the CPU on the same system and causes around four times as many bit flips as the CPU attack. We demonstrate the efficacy of JackHammer from the FPGA through a realistic fault attack on the WolfSSL RSA signing implementation that reliably causes a fault after an average of fifty-eight RSA signatures, $\mathbf{2 5 \%}$ faster than a CPU Rowhammer. In some scenarios our JackHammer attack produces faulty signatures more than three times more often and almost three times faster than a conventional CPU Rowhammer. Finally, we systematically analyze new cache attacks in these environments following demonstration of a cache covert channel across FPGA and CPU.
\end{abstract}

Keywords: FPGA · side-channel · cache attack · Rowhammer · cloud security

\section{Introduction}

In recent years, as improvements in the performance of microprocessors have slowed, developers have looked to other computing resources. FPGAs, graphics processing units (GPUs) and application-specific integrated circuits (ASICs) have all been adapted to accelerate applications such as high-frequency trading and machine learning. FPGAs are particularly interesting for cloud computing applications, as they can be reconfigured for the needs of different users at different times. Amazon Web Services [Ama17] and Alibaba Cloud [Ali19] already offer FPGA instances with ultra-high performance Xilinx Virtex UltraScale+ and Intel Arria 10 GX FPGAs to the consumer market. These FPGAs are designed for high I/O bandwidth and high compute capacity, making them ideal for server workloads. New Intel FPGAs offer cache-coherent memory systems for even better performance when data is being passed back and forth between CPU and FPGA. 
The flexibility of FPGA systems can also open up new attack vectors for malicious users in public clouds or more efficiently exploit existing ones. Integrated FPGA platforms connect the FPGA right into the processor bus interconnect giving the FPGA direct access into cache and memory [Huf18]. Similarly, high-end FPGAs can be integrated into a server as an accelerator, e.g. connected via PCIe interface [Int18b, Xil19]. Such combinations provide unprecedented performance over a high-throughput and low-latency connection with the versatility of a reprogrammable FPGA infrastructure shared among cloud users. However, the tight integration may also expose users to new adversarial threats.

This work exposes hardware vulnerabilities in hybrid FPGA-CPU systems with a particular focus on cloud platforms where the FPGA and the CPU are in distinct security domains: one potentially a victim and the other an attacker. We examine Intel's Arria 10 GX FPGA as an example of a current generation of FPGA accelerator platform designed in particular for heavy and/or cloud-based computation loads. We thoroughly analyze the memory interfaces between such platforms and their host CPUs. These interfaces, which allow the CPU and FPGA to interact in various direct and indirect ways, include hardware on both the FPGA and CPU, application libraries and software drivers executed by the CPU, and logical interfaces implemented on the FPGA outside of but accessible to the user-configurable region. We propose attacks that exploit practical use cases of these interfaces to target adjacent systems such as the CPU memory and cache.

\subsection{Our Contributions}

We demonstrate novel attacks between the memory interface of Intel Arria 10 GX platforms and their host CPUs. Furthermore, we demonstrate a Rowhammer mounted from the FPGA against the CPU to cause faults in the WolfSSL RSA signature implementation, and to leak a private RSA modulus factor. In summary:

- We thoroughly reverse-engineer and analyze the cache behavior and investigate the viability of cache attacks on realistic FPGA-CPU hybrid systems.

- Based on our study of the cache subsystem, we build JackHammer, a Rowhammer from the FPGA that bypasses caching to hammer the main memory. We compare JackHammer with the CPU Rowhammer and show that JackHammer is twice as fast as a CPU attack, causing faults that the CPU Rowhammer is unable to replicate. JackHammer remains stealthy to $\mathrm{CPU}$ monitors since it bypasses the $\mathrm{CPU}$ microarchitecture.

- Using both JackHammer and conventional CPU Rowhammer, we demonstrate a fault attack on recent versions of RSA implementation in the WolfSSL library and recover private keys. We show that the base blinding used in this RSA implementation leaves the algorithm vulnerable to the Bellcore fault injection attack.

- We systematically analyze cache attack techniques on different scenarios: FPGA to CPU, CPU to FPGA, and FPGA to FPGA, and demonstrate a cache covert channel that can transmit up to $1.5-1.8 \mathrm{MBit} / \mathrm{s}$ from the FPGA to the CPU.

Vulnerability Disclosure We informed the WolfSSL team about the vulnerability to Bellcore-style RSA fault injection attacks on November 25, 2019. WolfSSL acknowledged the vulnerability on the same day, and released WolfSSL 4.3.0 with a fix for the vulnerability on December 20, 2019. The vulnerability can be tracked via CVE-2019-19962 [mit19].

\subsection{Experimental Setup}

We experiment with two distinct FPGA-CPU platforms with Intel Arria 10 FPGAs: 1) integrated into the CPU package and 2) Programmable Acceleration Card (PAC). The integrated Intel Arria 10 is based on a prototype E5-2600v4 CPU with 12 physical cores. The CPU has a Broadwell architecture in which the last level cache (LLC) is inclusive of the L1/L2 caches. The CPU package has an integrated Arria 10 GX 1150 FPGA running 


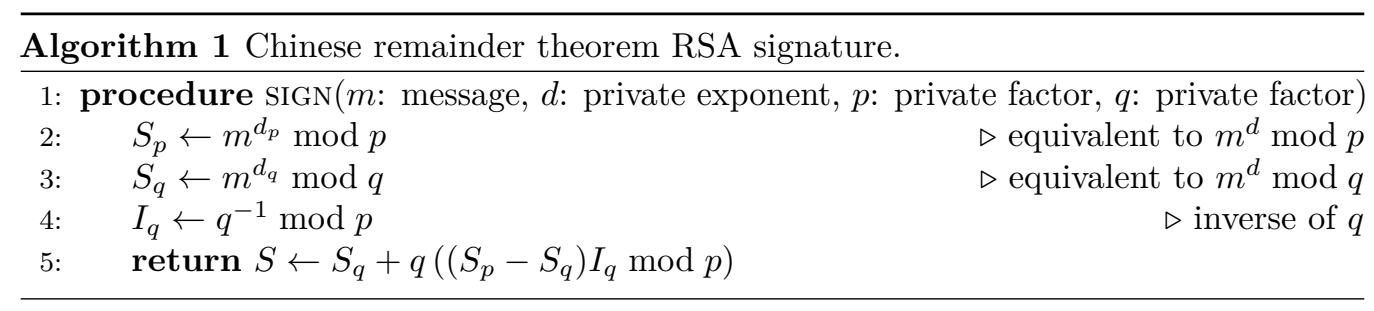

at $400 \mathrm{MHz}$. All measurements done on this platform are strictly done from userspace only, as access is kindly provided by Intel through their Intel Lab (IL) Academic Compute Environment. ${ }^{1}$ The IL environment also gives us access to platforms with PACs with Arria 10 GX 1150 FPGA installed and running at $200 \mathrm{MHz}$. These systems have Intel Xeon Platinum 8180 CPUs that come with non-inclusive LLCs. We carried out the Rowhammer experiments on our local Dell Optiplex 7010 system with an Intel i7-3770 CPU, and a single DIMM of Samsung M378B5773DH0-CH9 1333 MHz 2 GB DDR3 DRAM equipped with the same Intel PAC running with a primary clock speed of $200 \mathrm{MHz} .{ }^{2}$

The operating system (OS) running in the IL is a 64-bit Red Hat Enterprise Linux 7 with Kernel version 3.10. The OPAE version was compiled and installed on July 15th, 2019 for both the FPGA PAC and the integrated FPGA platform. We used Quartus 17.1.1 and Quartus 16.0.0 to synthesize AFUs for the PACs and integrated FPGAs, respectively. The bitstream version of the non-user-configurable Board Management Controller (BMC) firmware is 1.1.3 on the FPGA PAC and 5.0.3 on the integrated FPGA. The OS on our Optiplex 7010 workstation is Ubuntu 16.04.4 LTS with Linux kernel 4.13.0-36. On this system, we installed the latest stable release of OPAE, 1.3.0, and on its FPGA PAC, we installed the compatible 1.1.3 BMC firmware bitstream.

\section{Background}

\section{$2.1 \quad$ RSA-CRT Signing}

RSA signatures are computed by raising a plaintext $m$ to a secret power $d$ modulo $N=p q$, where $p$ and $q$ are prime and secret, and $N$ is public [BDL97]. These numbers must all be large for RSA to be secure, which makes the exponentiation rather slow. However, there is an algebraic shortcut for modular exponentiation: the Chinese Remainder Theorem (CRT), used in many RSA implementations, including in the WolfSSL we attack in Section 5 and in OpenSSL [CDFG18]. The basic form of the RSA-CRT signature algorithm is shown in Algorithm 1. The CRT algorithm is much faster than simply computing $m^{d} \bmod N$ because $d_{p}$ and $d_{q}$ are of order $p$ and $q$ respectively while $d$ is of order $N$, which, being the product of $p$ and $q$, is significantly greater than $p$ or $q$; it is around four times faster to compute the two exponentiations $m^{d_{p}}$ and $m^{d_{q}}$ than it is to compute $m^{d}$ outright $\left[\mathrm{ABF}^{+} 03\right]$.

\subsection{Cache Attacks}

Cache attacks have been proposed attacking various applications [OKSK15, BH09, GES17, GZES17, BvdPSY14, TSS ${ }^{+}$03]. In general, cache attacks use timing of the cache behavior to leak information. Modern cache systems use a hierarchical architecture that includes smaller, faster caches and bigger, slower caches. Measuring the latency of a memory access can often confidently determine which levels of cache contain a certain memory address (or if the memory is cached at all). Many modern cache subsystems also support

\footnotetext{
${ }^{1}$ https://wiki.intel-research.net/

${ }^{2}$ The PAC is intended to support $400 \mathrm{MHz}$ clock speed, but the current version of the Intel Acceleration Stack has a bug that halves the clock speed.
} 
coherency, which ensures that whenever memory is overwritten in one cache, copies of that memory in other caches are either updated or invalidated. Cache coherency may allow an attacker to learn about a cache line that is not even directly accessible [IES16]. Cache attacks have become a major focus of security research in cloud computing platforms where users are allocated CPUs, cores, or virtual machines which, in theory, should offer perfect isolation, but in practice may leak information to each other via shared caches [İGI $\left.{ }^{+} 16\right]$. An introduction to various cache attack techniques is given below:

Flush+Reload Flush+Reload (F+R) [YF14] has three steps: 1) The attacker uses the clflush instruction to flush the cache line that is to be monitored. After flushing this cache line, 2) she waits for the victim to execute. Later, 3) she reloads the flushed line and measures the reload latency. If the latency is low, the cache line was served from the cache hierarchy, so the cache line was accessed by the victim. If the access latency is high, the cache line was loaded from main memory, meaning that the victim did not access it. $\mathrm{F}+\mathrm{R}$ can work across cores and even across sockets, as long as the LLC is coherent, as is the case with many modern multi-CPU systems. Flush+Flush $(\mathrm{F}+\mathrm{F})$ [GMWM16] is similar to $\mathrm{F}+\mathrm{R}$, but the third step is different: the attacker flushes the cache line again and measures the execution time of the flush instruction instead of the memory access.

Orthogonal to $\mathrm{F}+\mathrm{R}$, if the attacker does not have access to an instruction to flush a cache line, she can instead evict the desired cache line by accessing cache lines that form an eviction set in an Evict+Reload $(\mathrm{E}+\mathrm{R})\left[\mathrm{LGS}^{+} 16\right]$ attack. Eviction sets are described shortly. $\mathrm{E}+\mathrm{R}$ can be used if the attacker shares the same CPU socket (but not necessarily the same core) as the victim and if the LLC is inclusive. ${ }^{3} \mathrm{~F}+\mathrm{R}, \mathrm{F}+\mathrm{F}$, and $\mathrm{E}+\mathrm{R}$ are limited to shared memory scenarios, where the victim and attacker share data or instructions, e.g. when memory de-duplication is enabled.

Prime+Probe Prime+Probe $(\mathrm{P}+\mathrm{P})$ gives the attacker less temporal resolution than the aforementioned methods since the attacker checks the status of the cache by probing a whole cache set rather than flushing or reloading a single line. However, this resolution is sufficient in many cases [OST06, RTSS09, ZJRR12, IES15a, OKSK15, LGS ${ }^{+}$16, MIE17]. $\mathrm{P}+\mathrm{P}$ has three steps: $\mathbf{1})$ The attacker primes the cache set under surveillance with dummy data by accessing a proper eviction set, 2) she waits for the victim to execute, 3) she accesses the eviction set again and measures the access latency (probing). If the latency is above a certain threshold, some parts of the eviction set was evicted by the victim process, meaning that the victim accessed cache lines belonging to the cache set under surveillance $\left[\mathrm{LYG}^{+} 15\right]$. Unlike $\mathrm{F}+\mathrm{R}, \mathrm{E}+\mathrm{R}$, and $\mathrm{F}+\mathrm{F}, \mathrm{P}+\mathrm{P}$ does not rely on shared memory. However, it is noisier, only works if the victim is located on the same socket as the attacker, and relies on inclusive caches. An alternative attack against non-inclusive caches is to target the cache directory structure $\left[\mathrm{YSG}^{+} 19\right]$.

Evict+Time In scenarios where the attacker can not probe the target cache set or line, but she can still influence the target cache line, an Evict+Time $(\mathrm{E}+\mathrm{T})$ is still possible depending on the target application. In an E+T attack, the attacker only evicts the victim's cache line and measures the aggregate execution time of the victim's operation, hoping to observe a correlation between the execution time of an operation such as a cryptographic routine and the cache access pattern.

Eviction Sets Caches store data in units of cache lines that can hold $2^{b}$ bytes each (64 bytes on Intel CPUs). Caches are divided into $2^{s}$ sets, each capable of holding $w$ cache lines. $w$ is called the way-ness or associativity of the cache. An eviction set is a set of congruent cache line addresses capable of filling a whole cache set. Two cache lines are considered congruent if they belong to the same cache set. Memory addresses are mapped to cache sets depending on the $s$ bits of the physical memory address directly following the

\footnotetext{
${ }^{3} \mathrm{~A}$ lower-level cache is called inclusive of a higher-level cache if all cache lines present in the higher-level cache are always present in the lower-level cache.
} 
$b$ cache line offset bits, which are the least significant bits. Additionally, some caches are divided into $n$ slices, where $n$ is the number of CPU cores. In the presence of slices, each slice has $2^{s}$ sets with $w$ ways each. Previous work has reverse-engineered the mapping of physical address bits to cache slices on some Intel processors [IES15b]. A minimal eviction set contains $w$ addresses and therefore fills an entire cache set when accessed.

\subsection{Rowhammer}

DRAM cells discharge over time, and the memory controller has to refresh the cells to avoid accidental data corruption. Generally, DRAM cells are laid out in banks and rows, and each row within a bank has two adjacent rows, one on either side. In a Rowhammer attack, memory addresses in the same bank as the target memory address are accessed in quick succession. When memory adjacent to the target is accessed repeatedly, the electrostatic interference generated by the physical process of accessing the memory can elevate the discharge for bits stored in the target memory. A "single-sided" Rowhammer performs accesses to just one of these rows to generate bit flips in the target row; a "double-sided" Rowhammer performs accesses to both adjacent rows and is generally more effective in producing bit flips. Rowhammer relies on the ability to find blocks of memory accessible to the malicious program (or in this work, hardware) that are in the same memory bank as a given target address. The standard way of finding these memory addresses is by exploiting row buffer conflicts as a timing side-channel [FGBR18]. Pessl et al. [ $\left.\mathrm{PGM}^{+} 16\right]$ reverse-engineered the bank mapping algorithms of several CPU and DRAM configurations which allows an attacker to deterministically calculate all of the physical addresses that share the same bank if the chipset and memory configuration are known.

\subsection{Related Attacks}

Classical power analysis techniques like Kocher et al.'s differential power analysis [KJJ99] have been applied in new attacks on inter-chip FPGAs [ZS18, SGMT18b, SGMT18a]. Such integrated and inter-chip FPGAs are available in various cloud environments and system-on-chips (SoCs) products. In particular, Zhao et al. [ZS18] demonstrated how to build an on-chip power monitor using ring oscillators (ROs) which can be used to attack the host CPU or other FPGA tenants. In multi-tenant FPGA scenarios where partial reconfiguration by two separate security domains is possible, more powerful attacks become possible. For instance, the long wires on the FPGA can spy on adjacent wires using ROs [GER19, RPD $\left.{ }^{+} 18, \mathrm{PRP}^{+} 19\right]$. Ramesh et al. $\left[\mathrm{RPD}^{+} 18\right]$ exploited the speed of ROs to infer the carried bit in the adjacent wire and demonstrated a key recovery attack on AES. ROs can also be used as power wasters to create voltage drop and timing faults [GOT17, KGT18]. Note that such attacks rely on FPGA multi-tenancy which is not widely used yet. In contrast, in this work, we only assume that the FPGA-CPU memory subsystem is shared among tenants.

\section{Analysis of Intel FPGA-CPU Systems}

This section explains the hardware and software interfaces that the Intel Arria 10 GX FPGA platforms use to communicate with their host CPUs and the firmware, drivers, and architectures that underlay them. Figure 1 gives an overview of such architecture.

Introduction to Intel Terminology Intel refers to a single logical unit implemented in FPGA logic and having a single interface to the CPU as an Accelerator Functional Unit (AFU). So far, available FPGA platforms only support one AFU per Partial Reconfiguration Unit (PRU, also called the Green Region). The AFU is an abstraction similar to a program that captures the logic implemented on an FPGA. The FPGA Interface Manager (FIM) is 


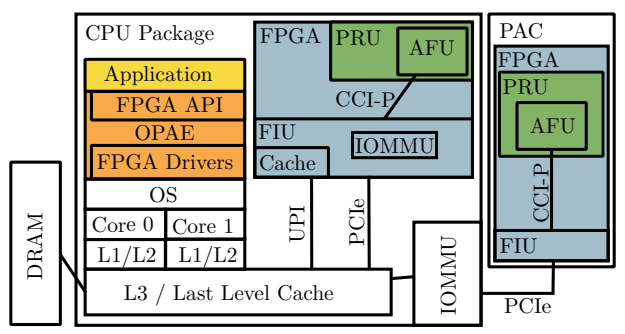

Figure 1: Overview of the architecture of Intel FPGAs. The software part of the Intel Acceleration Stack called OPAE is highlighted in orange. Its API is used by applications (yellow) to communicate with the AFU. The Green Region marks the part of the FPGA that is re-configurable from userspace at runtime. The Blue Region describes the static soft core of the FPGA. It exposes the CCI-P interface to the AFU.

part of the non-user-configurable portion (Blue Region) of the FPGA and contains external interfaces like memory and network controllers as well as the FPGA Interface Unit (FIU), which bridges those external interfaces with internal interfaces to the AFU.

\subsection{Intel FPGA Platforms}

Intel's Arria 10 GX Programmable Acceleration Card (PAC) is a PCIe expansion card for FPGA acceleration [Int18b]. The Arria 10 GX FPGA on the card communicates with its host processor over a single PCIe Gen3x8 bus. Memory reads and writes from the FPGA to the CPU's main memory use physical addresses; in virtual environments, the PCIe controller on the CPU side implements an I/O memory management unit (IOMMU) to translate physical addresses in the virtual machine (what Intel calls I/O Virtual Addresses or IOVA) to physical addresses in the host. Alongside the FPGA, the PAC carries 8 GB of DDR4, $128 \mathrm{MB}$ of flash memory, and USB for debugging.

An alternative accelerator platform is the Xeon server processor with an integrated Arria 10 FPGA in the same package [Huf18]. The FPGA and CPU are closely connected through two PCIe Gen3x8 links and an UltraPath Interconnect (UPI) link. UPI is Intel's high-speed CPU interconnect (replacing its predecessor QPI) in Skylake and later Intel CPU architectures [Mul17]. The FPGA has a $128 \mathrm{KiB}$ directly mapped cache that is coherent with the CPU caches over the UPI bus. Like the PCIe link on the PAC, both the PCIe links and the UPI link use I/O virtual addressing, appearing as physical addresses to virtualized environments. As the UPI link bypasses the PCIe controller's IOMMU, the FIU implements its own IOMMU and Device TLB to translate physical addresses for reads and writes using UPI [Int18a].

\subsection{Intel's FPGA-CPU Compatibility Layers}

Open Programmable Acceleration Engine (OPAE) Intel's latest generations of FPGA products are designed for use with the OPAE [Int17] which is part of the Intel Acceleration Stack. The principle behind OPAE is that it is an open-source, hardwareflexible software stack designed for interfacing with FPGAs that use Intel's Core Cache Interface (CCI-P), a hardware host interface for AFUs that specifies transaction requests, header formats, timing, and memory models [Int18a]. OPAE provides a software interface for software developers to interact with a hosted FPGA, while CCI-P provides a hardware interface for hardware developers to interact with a host CPU. Excluding a few platformspecific hardware features, any CCI-P compatible AFU should be synthesizable (and the result should be logically identical) for any CCI-P compatible FPGA platform; OPAE is built on top of hardware- and OS-specific drivers and as such is compatible with any 
Table 1: Overview of the caching hints configurable over CCI-P on an integrated FPGA. *_I hints invalidate a cache line in the local cache. Reading with RdLine_S stores the cache line in the shared state. Writing with WrLine_M caches the line modified state.

\begin{tabular}{l|lllll}
\hline Cache Hint & RdLine_I & RdLine_S & WrLine_I & WrLine_M & WrPush_I \\
\hline Desc. & $\begin{array}{l}\text { No FPGA } \\
\text { caching }\end{array}$ & $\begin{array}{l}\text { Leave FPGA } \\
\text { cache in S state }\end{array}$ & $\begin{array}{l}\text { No FPGA } \\
\text { caching }\end{array}$ & $\begin{array}{l}\text { Leave FPGA } \\
\text { cache in M state }\end{array}$ & $\begin{array}{l}\text { Intent to cache } \\
\text { in LLC }\end{array}$ \\
\hline Available & UPI, PCIe & UPI & UPI, PCIe & UPI & UPI, PCIe
\end{tabular}

system with the appropriate drivers available. As described below, the OPAE/CCI-P system provides two main methods for passing data between the host CPU and the FPGA.

Memory-mapped I/O (MMIO) OPAE can send 32- or 64-bit MMIO requests to the AFU directly or it can map an AFU's MMIO space to OS virtual memory [Int17]. CCI-P provides an interface for incoming MMIO requests and outgoing MMIO read responses. The AFU may respond to read and write requests in any way that the developer desires, though an MMIO read request will time out after 65,536 cycles of the primary FPGA clock. In software, MMIO offsets are counted as the number of bytes and expected to be multiples of 4 (or 8 , for 64-bit reads and writes), but in $\mathrm{CCl}-\mathrm{P}$, the last two bits of the address are truncated, because at least 4 bytes are always being read or written. There are 16 available address bits in CCI-P, meaning that the total available MMIO space is $2^{16}$ 32-bit words, or $256 \mathrm{KiB}$ [Int18a].

Direct memory access (DMA) OPAE can request the OS to allocate a block of memory that can be read by the FPGA. There are a few important details in the way this memory is allocated: most critically, it is allocated in a contiguous physical address space. The FPGA will use physical addresses to index the shared memory, so physical and virtual offsets within the shared memory must match. On systems using Intel Virtualization Technology for Directed I/O (VT-d), which employs the IOMMU to provide an IOVA to PCIe devices, the memory will be allocated in continuous IOVA space. Either way, this ensures that the FPGA will see an accessible and continuous buffer of the requested size. For buffer sizes up to and including one $4 \mathrm{kB}$ memory page, a normal memory page will be allocated to the calling process by the OS and configured to be accessible by the FPGA with its IOVA or physical address. For buffer sizes greater than $4 \mathrm{kB}$, OPAE will call the OS to allocate a $2 \mathrm{MB}$ or $1 \mathrm{~GB}$ huge page. Keeping the buffer in a single page ensures that it will be contiguously allocated in physical memory.

\subsection{Cache and Memory Architecture on the Intel FPGAs}

Arria 10 PAC The Arria 10 PAC has access to the CPU's memory system as well as its own local DRAM with a separate address space from that of the CPU and its memory. The PAC's local DRAM is always directly accessed, without a separate caching system. When the PAC reads from the CPU's memory, the CPU's memory system will serve the request from its LLC if possible. If the memory that is read or written is not present in the LLC, the request will be served by the CPU's main DRAM. The PAC is unable to place cache lines into the LLC with reads, but writes from the PAC update the LLC.

Integrated Arria 10 The integrated Arria 10 FPGA has access to the host memory. Additionally, it has its own $128 \mathrm{kB}$ cache that is kept coherent with the CPU's caches over UPI. Memory requests over PCIe take the same path as requests issued by an FPGA PAC. If the request is routed over UPI, the local coherent FPGA cache is checked first, on a cache miss, forwarding the request to the CPU's LLC or main memory. 


\subsubsection{Reverse-engineering Caching Hint Behavior}

An AFU on the Arria $10 \mathrm{GX}$ can exercise some control over caching behavior by adding caching hints to memory requests. The available hints are summarized in Table 1. For memory reads, RdLine_I is used to not cache data locally and RdLine_S to cache data locally in the shared state. For memory writes, WrLine_I is used to prevent local caching on the FPGA, WrLine_M leaves written data in the local cache in the modified state. WrPush_I does not cache data locally but hints the cache controller to cache data in the CPU's LLC. The CCI-P documentation lists all caching hints as available for memory requests over UPI [Int18a]. When sending requests over PCI, only RdLine_I, WrLine_I, and WrPush_I can be used while other hints are ignored. However, based on our experiments, not all cache hints are implemented exactly to specification.

To confirm the behavior of caching hints available for DMA writes, we designed an AFU that writes a constant string to a configurable memory address using a configurable caching hint and bus. We used the AFU to write a cache line and afterward timed a read access to the same cache line on the CPU. These experiments confirm that nearly $100 \%$ of the cache lines written to by the AFU are placed in the LLC, as access times stay below $100 \mathrm{CPU}$ clock cycles while main memory accesses take 175 cycles on average. This behavior is independent of the caching hint, the bus, or the platform (PAC, integrated Arria 10). The result is surprising as the caching hint meant to cache the data in the cache of the integrated Arria 10 and the caching hint meant for writing directly to the main memory are either ignored by the Blue Region and the CPU or not implemented yet. Intel later verified that the Blue Region in fact ignores all caching hints that apply to DMA writes. Instead, the CPU is configured to handle all DMA writes as if the WrPush_I caching hint is set. The observed LLC caching behavior is likely caused by Intel's Data Direct I/O (DDIO), which is enabled by default in recent Intel CPUs. DDIO is meant to give peripherals direct access to the LLC and thus causes the CPU to cache all memory lines written by the AFU. DDIO restricts cache access to a subset of ways per cache set, which reduces the attack surface for Prime+Probe attacks. Nonetheless, attacks against other DDIO-enabled peripherals are possible [TVT19, $\left.\mathrm{KGA}^{+} 19\right]$.

\section{JackHammer Attack}

Contribution In this section, we present and evaluate a simple AFU for the Arria $10 \mathrm{GX}$ FPGA that is capable of performing Rowhammer against its host CPU's DRAM as much as two times faster and four times more effectively than its host CPU can. In a Rowhammer, a significant factor in the speed and efficacy of an attack is the rate at which memory can be repeatedly accessed. On many systems, the CPU is sufficiently fast to cause some bit flips, but the FPGA can repeatedly access its host machine's memory system substantially faster than the host machine's CPU can. Both the CPU and FPGA share access to the same memory controller, but the CPU must flush the memory after each access to ensure that the next access reaches DRAM; memory reads from the FPGA do not affect the CPU cache system so no time is wasted flushing memory with the FPGA implementation. We further measure the performance of CPU and FPGA Rowhammer implementations with caching both enabled and disabled, and find that disabling caching brings CPU Rowhammer speed near that of our FPGA Rowhammer implementation. Crucially, the architectural difference also means that it is much more difficult for a program on the CPU to detect the presence of an FPGA Rowhammer than that of a CPU Rowhammer the FPGA's memory accesses leave far fewer traces on the CPU. 


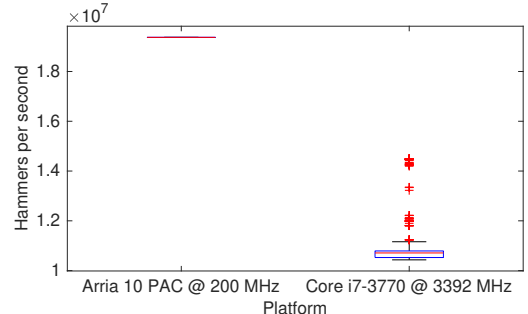

Figure 2: Distributions of hammering rates (memory requests per second) on FPGA PAC and i7-3770.

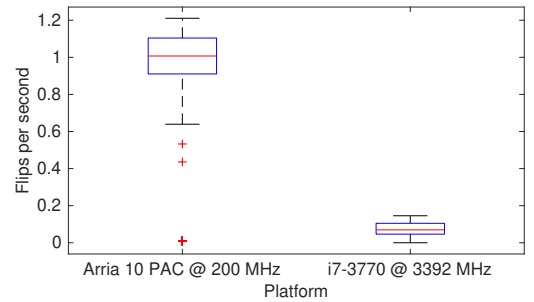

Figure 3: Distributions of flip rates on FPGA PAC and i7-3770.

\subsection{JackHammer: Our FPGA Implementation of Rowhammer}

JackHammer supports configuration through the MMIO interface. When the JackHammer AFU is loaded, the CPU first sets the target physical addresses that the AFU will repeatedly access. It is recommended to set both addresses for a double-sided attack, but if the second address is set to 0 , JackHammer will perform a single-sided attack using just the first address. The CPU must also set the number of times to access the targeted addresses.

When the configuration is set, the CPU signals the AFU to repeat memory accesses and issue them as fast as it can, alternating between addresses in a double-sided attack. Note that unlike a software implementation of Rowhammer, the accessed addresses do not need to be flushed from cache - DMA read requests from the FPGA do not cache the cache line in the CPU cache, though if the requested memory is in the last-level cache, the request will be served to the FPGA by the cache instead of by memory (see Section 3.3 for more details on caching behavior). In this attack, the attacker needs to ensure that the cache lines used for inducing bit flips are never accessed by the CPU during the attack. The number of times to access the target addresses can be read again to get the number of remaining accesses; this is the simplest way to check in software whether or not the AFU has finished sending these accesses. When the last read request has been sent by the AFU, the total amount of time taken to send all of the requests is recorded. ${ }^{4}$

\subsection{JackHammer on the FPGA PAC vs. CPU Rowhammer}

Figure 2 shows a box plot of the 0th, 25th, 50th, 75th, and 100th percentile of measured "hammering rates" on the Arria 10 FPGA PAC and its host i7-3770 CPU. Each measurement in these distributions is the average hammering rate over a run of 2 billion memory requests. Our JackHammer implementation is substantially faster than the standard CPU Rowhammer, and its speed is far more consistent than the CPU's. The FPGA can manage an average throughput of one memory request, or "hammer," every ten $200 \mathrm{MHz}$ FPGA clock cycles (finishing 2 billion hammers in an average of 103.25 seconds); the CPU averages one hammer every $3113.4 \mathrm{GHz}$ CPU clock cycles (finishing 2 billion hammers in an average of 183.41 seconds). Here we can see that even if the FPGA were clocked higher, it would still spend most of its time waiting for entries in the PCIe transaction buffer in the non-reconfigurable region to become available.

Figure 3 shows measured bit flip rates in the victim row for the same experiment. Runs where zero flips occurred during hardware or software hammering were excluded from the flip rate distributions, as they are assumed to correspond with sets of rows that are in

\footnotetext{
${ }^{4}$ The time to send all the requests is not precisely the time to complete all the requests, but it is very close for sufficiently high numbers of requests. The FPGA has a transaction buffer that holds up to 64 transactions after they have been sent by the AFU. The buffer does take some time to clear, but the additional time is negligible for our performance measurements of millions of requests.
} 


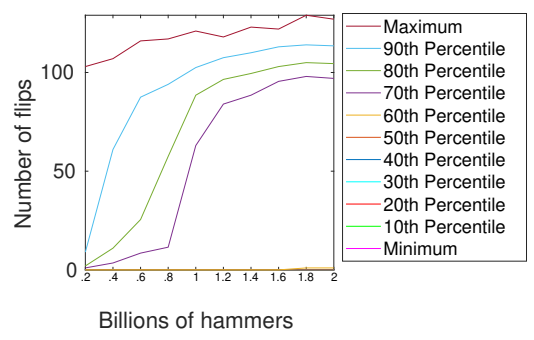

Figure 4: Distributions of total flips after 200 million to 2 billion hammers on PAC.

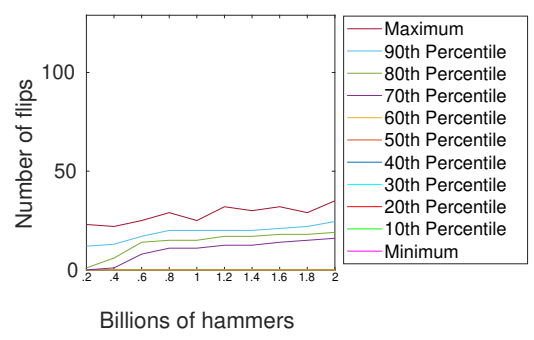

Figure 5: Distributions of total flips after 200 million to 2 billion hammers on i7-3770.

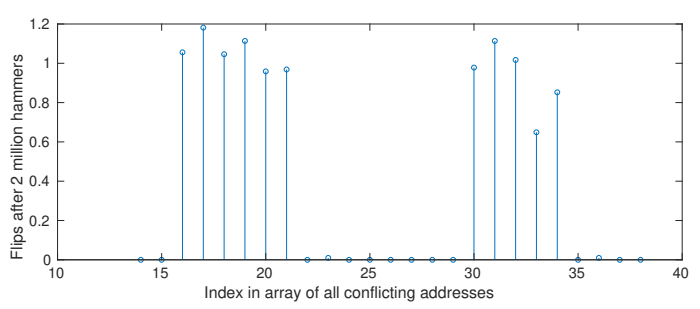

Figure 6: Time series plotting number of flips on a row-by-row basis, showing an example of the consistent placement of small-valued outliers (samples 23 and 36 on this graph) relative to their much larger neighbors. These rows only ever incur a few flips, compared to most "flippy" rows which incur dozens of flips, and always are located two rows away from a block of rows that flip much more.

the same logical bank, but not directly adjacent to each other. The increased hammering speed of JackHammer produces a more than proportional increase in flip rate, which is unsurprising due to the highly nature of Rowhammer. As the Rowhammer is underway, electrical charge is drained from capacitors in the victim row. However, the memory controller also periodically refreshes the charge in the capacitors. When there are more memory accesses to adjacent rows within each refresh window, it is more likely that a bit flip occurs before the next refresh. This is why the FPGA's increased memory throughput is more effective for conducting Rowhammer against the same DRAM chip.

Another way to look at hammering performance is by counting the total number of flips produced by a given number of hammers. Figure 4 and Figure 5 show minimum, maximum, and every 10th percentile of the number of flips produced by the AFU and CPU respectively for a range of total number of hammers from 200 million to 2 billion. These graphs demonstrate how much more effectively the FPGA PAC can generate bit flips in the DRAM even after the same number of memory accesses. For hammering attempts that resulted in a non-zero number of bit flips, the AFU exhibits a wide distribution of flip count in the range of 200 million to 800 million hammers which then rapidly narrows in the range of 800 million to 1.2 billion and finally levels out by 1.8 billion hammers. This set of distributions seems to indicate that "flippable" rows will ultimately reach about 80-120 total flips after enough hammering, but it can take anywhere from 200 million hammers (about 10 seconds) to 2 billion hammers (about 100 seconds) to reach that limit.

There are also a few rows that only incur a few flips. These samples appear in a consistent pattern demonstrated in Figure 6, which plots a portion of the data used to create Figure 4 in detail. Each impulse in this plot represents the number of flips after a single run of 2 billion hammers on a particular target row. In Figure 6, at indices 23 and 36 , two of these outliers are visible, each appearing two indices after several samples in the standard 80-120 flip range. These outliers could indicate rows that are affected vary slightly by hammering on rows that are nearby but not adjacent. 


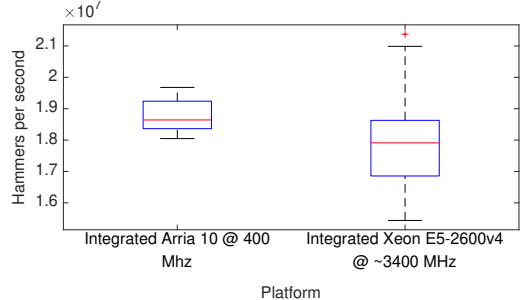

Figure 7: Distributions of hammering rates (memory requests per second) on integrated Arria 10 and Xeon E5-2600 v4.

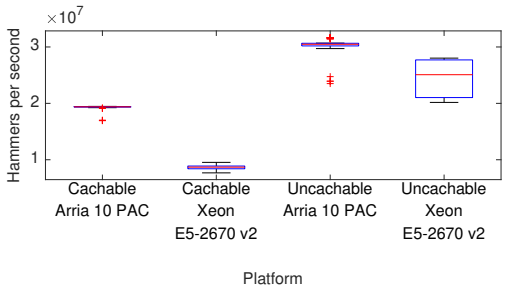

Figure 8: Distributions of hammering rates (memory requests per second) with cachable and uncachable memory.

\subsection{JackHammer on the Integrated Arria 10 vs. CPU Rowhammer}

The JackHammer AFU we designed for the integrated platform is the same as the AFU for the PAC, except that the integrated platform has access to more physical channels for the memory reads. The PAC only has a single PCIe channel; the integrated platform has one UPI channel and two PCIe channels, as well as an "automatic" setting which lets the interface manager select a physical channel automatically. Therefore we present the hammering rates on this platform with two different settings - alternating PCIe lanes on each access and using the automatic setting.

However, this platform is only available to us on Intel's servers, so we have only been able to test on one DRAM setup and have been unable to get bit flips on this DRAM. ${ }^{5}$ The integrated Arria 10 shares the package with a modified Xeon v4-style CPU. The available servers are equipped with an X99 series motherboard with 64 GB of DDR4 memory. Figure 7 shows distributions of measured hammering rates on the integrated Arria 10 platform. Compared to the Arria 10 PAC, the integrated Arria 10's hammering rate is more varied, but with a similar mean rate.

\subsection{The Effect of Caching on Rowhammer Performance}

We hypothesized that a primary reason for the difference in Rowhammer performance between JackHammer on the FPGAs and a typical Rowhammer implementation on the CPUs is that when one of the FPGAs reads a line of memory from DRAM, it is not cached, so the next read will miss the cache and be directed to the DRAM as well. On the other hand, when the CPUs access a line of memory, it is cached, and the memory line must be flushed from cache before the next read is issued, or the next read will hit the cache instead of DRAM, and the physical row in the DRAM will not be "hammered."

To evaluate our hypothesis that caching is an important factor in the performance disparity we observed between FPGA- and CPU-based Rowhammer, we used the PTEditor [Sch19] kernel module to set allocated pages as uncachable before testing hammering performance. We edited the setup of the Rowhammer performance tests to allocate many $4 \mathrm{kB}$ pages and set all of those as uncachable instead of one $2 \mathrm{MB}$ huge page, as the kernel module we used to set the pages as uncachable was not correctly configuring the huge pages as uncachable. However, it is still easy to find a large continuous range of physical addresses — when these pages are allocated by OPAE, the physical address is directly available to the software. So the software simply allocates thousands of $4 \mathrm{kB}$ pages, sorts them, and

\footnotetext{
${ }^{5}$ There are several reasons why this could be the case. Some DRAM is simply more resistant to Rowhammer by its physical nature. DDR4 memory, which can be found in this system, sometimes has hardware features to block Rowhammer style attacks [JC-17]; though some methods have been developed to circumvent these protections $\left[\mathrm{GLS}^{+} 18\right]$, these methods ultimately still rely on the ability of the attacker to repeatedly access the DRAM very quickly, so we consider those methods outside of the scope of this research, which is focused on the relative ability of the FPGA platforms to quickly access DRAM.
} 


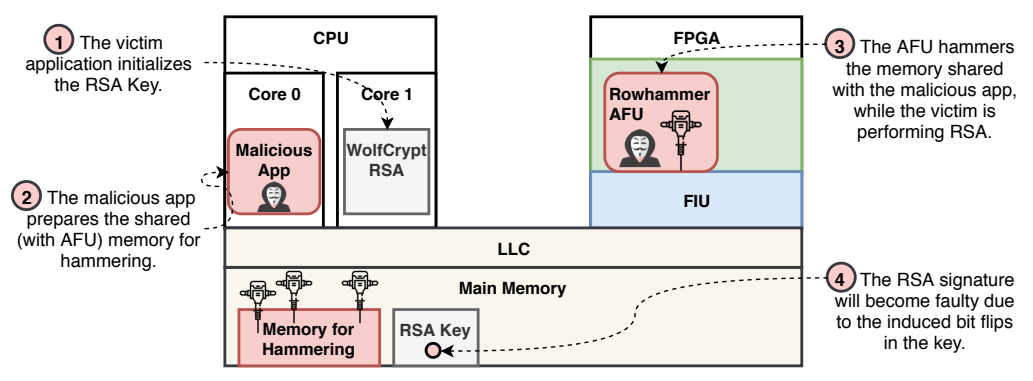

Figure 9: WolfSSL RSA Fault Injection Attack.

then finds the biggest continuous range within them and attempts to find colliding row addresses within that range. The JackHammer AFU required no modifications from the initial performance tests; the assembly code to hammer from the CPU was edited to not flush the memory after reading it, since the memory will not be cached in the first place.

We performed this experiment by placing the FPGA PAC on a Dell Poweredge R720 system with a Xeon E5-2670 v2 CPU fixed to a clock speed of $2500 \mathrm{MHz}$ and two $4 \mathrm{~GB}$ DIMMs of DDR3 DRAM clocked at $1600 \mathrm{MHz}$. Figure 8 shows the performance of the FPGA PAC and this system's CPU with caching enabled and disabled. Disabling caching produces a significant speedup in hammering for both the PAC and the CPU, but especially for the CPU, which saw a $188 \%$ performance increase. With caching enabled, the median hammering rate of the PAC was more than twice that of the CPU, but with caching disabled, the median hammering rate of the PAC was only $22 \%$ faster than that of the CPU. Of course, memory accesses on modern systems are extremely complex (even with caching disabled), so there are likely some factors affecting the changes in hammering rate that we cannot describe, but our experimental evidence supports our hypothesis that time spent flushing the cache is a major factor slowing down CPU Rowhammer implementations compared to FPGA implementations.

\section{Fault Attack on RSA using JackHammer}

Rowhammer has been used for fault injections on cryptographic schemes [BM16, BM18] or for privilege escalation [GLS ${ }^{+} 18, \mathrm{vdVFL}^{+} 16$, SD15]. Using JackHammer, we demonstrate a practical fault injection attack from the Arria 10 FPGA to the WolfSSL RSA implementation running on its host CPU. In the RSA fault injection attack proposed by Boneh et al. [BDL97], an intermediate value in the Chinese remainder theorem modular exponentiation algorithm is faulted, causing an invalid signature to be produced. Similarly, we attack the WolfSSL RSA implementation using JackHammer from the FPGA PAC and Rowhammer from the host CPU, and compare the efficiency of the two attacks. The increased hammering speed and flip rate of the Arria 10 FPGA makes the attack more practical in the time frame of about 9 RSA signatures.

Figure 9 shows the high-level overview of our attack: the WolfSSL RSA application runs on one core, while a malicious application runs adjacent to it, assisting the JackHammer AFU on the FPGA in setting up the attack. JackHammer causes a hardware fault in the main memory, and when the WolfSSL application reads the faulty memory, it produces a faulty signature and leaks the private factors used in the RSA scheme.

\subsection{RSA Fault Injection Attacks}

We implement a fault injection attack against the Chinese remainder theorem implementation of the RSA algorithm, commonly known as the Bellcore attack [BDL97]. Algorithm 1 
shows the Chinese remainder theorem (CRT) RSA signing scheme where the signature $S$ is computed by raising a message $m$ to the private exponent $d$ th power, modulo $N . d_{p}$ and $d_{q}$, are precomputed as $d \bmod p-1$ and $d \bmod q-1$, where $p$ and $q$ are the prime factors of $N$ [ $\left.\mathrm{ABF}^{+} 03\right]$. When one of the intermediates $S_{q}$ or $S_{p}$ is computed incorrectly, an interesting case arises. Consider the difference between a correctly computed signature $S$ of a message $m$ and an incorrectly computed signature $S^{\prime}$ of the same message, computed with an invalid intermediate $S_{p}^{\prime}$. The difference $S-S^{\prime}$ leaves a factor of $q$ times the difference $S_{p}-S_{p}^{\prime}$, so the GCD of $S-S^{\prime}$ and $N$ is the other factor $p\left[\mathrm{ABF}^{+} 03\right]$. This reduces the problem of factoring $N$ to a simple subtraction and GCD operation, so the private factors $(p, q)$ are revealed if the attacker has just one valid signature and one faulty signature, each signed on the same message $m$. These factors can also be recovered with just one faulty signature if the message $m$ and public key $e$ are known; it is also equal to the GCD of $S^{\prime e}-m$ and $N$.

Fault Injection Attack with RSA Base Blinding A common modification to any RSA scheme is the addition of base blinding, effective against simple and differential power analysis side-channel attacks, but vulnerable to a correlational power analysis attack demonstrated by [WvWM11]. Base blinding is used by default in our target WolfSSL RSA-CRT signature scheme. In this blinding process, the message $m$ is blinded by a randomly generated number $r$ by computing $m_{b}=m \cdot r^{e} \bmod n$. The resulting signature $S_{b}=\left(m \cdot r^{e}\right)^{d} \bmod n=m^{d} \cdot r \bmod n$ must then be multiplied by the inverse of the random number $r$ to generate a valid signature $S=S_{b} \cdot r^{-1} \bmod n$.

This blinding scheme does not prevent against the Bellcore fault injection attack. Consider a valid signature blinded with random factor $r_{1}$ and an invalid signature blinded with $r_{2}$. When the faulty signature is subtracted by the valid signature, the valid and blinded intermediates $S_{p b}$ are each unblinded and cancel as before, as shown in Equation 1.

$$
\begin{aligned}
S-S^{\prime}= & {\left[S_{q b}+q \cdot\left(\left(S_{p b}-S_{q b}\right) \cdot q^{-1} \bmod p\right)\right] \cdot r_{1}^{-1} \bmod N } \\
& -\left[S_{q b}+q \cdot\left(\left(S_{p b}^{\prime}-S_{q b}\right) \cdot q^{-1} \bmod p\right)\right] \cdot r_{2}^{-1} \bmod N \\
= & q \cdot\left[\left(S_{p b} \cdot q^{-1} \bmod p\right) \cdot r_{1}^{-1}-\left(S_{p b}^{\prime} \cdot q^{-1} \bmod p\right) \cdot r_{2}^{-1}\right] \bmod N
\end{aligned}
$$

Ultimately, there is still a factor of $q$ in the the difference $S-S^{\prime}$ which can be extracted with a GCD as before.

\subsection{Our Attack}

Approach and Justification We developed a simplified attack model to test the effectiveness of the Arria 10 Rowhammer in a fault injection scenario. Our model simplifies the setup of the attack so that we can efficiently measure the performance of both CPU Rowhammer and JackHammer. We sign the same message with the same key repeatedly while the Rowhammer exploit runs, and count the number of correct signatures until a faulty signature is generated, which is used to leak the private RSA key.

Attack Setup In summary, our simplified attack model works as follows: The attacker first allocates a large block of memory and checks it for conflicting row addresses. It then quickly tests which of those rows can be faulted with hammering using JackHammer. A list of rows that incur flips is saved so that it can be iterated over. The program then begins the "attack," iterating through each row that incurred flips during the test, and through the sixty-four 1024-bit offsets that make up the row. During the attack, the JackHammer $\mathrm{AFU}$ is instructed to repeatedly access the rows adjacent to the target row. Meanwhile, in the "victim" program, the targeted data (the precomputed intermediate value $d \bmod q-1$ ) is copied to the target address, which is computed as an offset of the targeted row. The victim then enters a loop where it reads back the data from the target row and uses it as part of an RSA key to create a signature from a sample message. Additionally, the 
"attacker" opens a new thread on the CPU which repeatedly flushes the target row on a given interval. It is necessary for the attacker to flush the target row because the victim is repeatedly reading the targeted data and placing it in cache, but the fault will only ever occur in main memory. For the victim program to read the faulty data from DRAM, there cannot be an unaffected copy of the same data in cache or the CPU will simply read that copy. As we show below, the performance of the attack depends significantly on the time interval between flushes.

One of the typical complications of a Rowhammer fault injection attack is ensuring that the victim's data is located in a row that can be hammered. In our simplified model, we choose the location of the victim data manually within a row that we have already determined to be one that incurs flips under a Rowhammer attack so that we may easily test the effectiveness of the attack at various rows and various offsets within the rows. In a real attack, the location of the victim program's memory can be controlled by the attacker with a technique known as page spraying [SD15], which is simply allocating a large number of pages and then deallocating a select few, filling the memory in an attempt to cause the victim program to allocate the right pages. Improvements in this process can be made; for example, [BM16] demonstrated how cache attacks can be used to gather information about the physical addresses of data being used by the victim process.

The other simplification in our model is that we force the CPU to read from DRAM using the clflush instruction to flush the targeted memory from cache. In an end-to-end attack, the attacker would use an eviction set to evict the targeted memory since it is not directly accessible in the attack process's address space. However, the effect is ultimately the same - the targeted data is forcibly removed from the cache by the attacker.

\subsection{Performance of the Attack}

In this section, we show that our JackHammer implementation with optimal settings can cause a faulty signature an average of $17 \%$ faster than a typical CPU-based, software-driven Rowhammer implementation with optimal settings. In some scenarios, the performance is as much as 4.8 times that of the software implementation. However, under some conditions, the software implementation can be more likely to cause a fault over a longer period of time. Our results indicate that increasing the DRAM row refresh rate provides significant but not complete defense against both implementations.

The performance of this fault injection attack is highly dependent on the time interval between evictions, and as such we present all of our results in this section as functions of the eviction interval. Each eviction triggers a subsequent reload from memory when the key is read for the next signature, which refreshes the capacitors in the DRAM. Whenever DRAM capacitors are refreshed, any accumulated voltage error in each capacitor (due to Rowhammer or any other physical effect) is either solidified as a new faulty bit value or reset to a safe and correct value. Too short of an interval between evictions will cause the DRAM capacitors to be refreshed too quickly to be flipped with a high probability. On the other hand, however, longer intervals can mean the attack is waiting to evict the memory for a longer time while a bit flip has already occurred. It is crucial to note, also, that DRAM capacitors are automatically refreshed by the memory controller on a $64 \mathrm{~ms}$ interval $^{6}$ [GMM16]. On some systems, this interval is configurable: faster refresh rates reduce the rate of memory errors, including those induced by Rowhammer, but they can impede maximum performance because the memory spends more time doing maintenance refreshes rather than serving read and write request. For more discussion on modifying row refresh rates as a defense against Rowhammer, see Section 7.

In Table 2 we present two metrics with which we compare JackHammer and a standard CPU Rowhammer implementation. This table shows the mean number of signatures until

\footnotetext{
${ }^{6}$ More specifically, DDR3 and DDR4 specifications indicate $64 \mathrm{~ms}$ as the maximum allowable time between DRAM row refreshes.
} 
Table 2: Performance of our JackHammer exploit compared to a standard software CPU Rowhammer with various eviction intervals. JackHammer is able to acheive better performance in many cases because it bypasses caching architecture, sending more memory requests during the eviction interval and causing bit flips at a higher rate.

\begin{tabular}{r|ccccccc}
\hline \multirow{2}{*}{$\begin{array}{r}\text { Eviction } \\
\text { Interval }\end{array}$} & \multicolumn{3}{|c}{ Mean signatures to fault } & & \multicolumn{3}{c}{ Successful fault rate } \\
\cline { 2 - 3 } & CPU & JackHammer & \% Inc. Speed & & CPU & JackHammer & \% Inc. Rate \\
\hline 16 & 280 & 186 & $51 \%$ & & $0.4 \%$ & $0.2 \%$ & $-46 \%$ \\
32 & 627 & 219 & $185 \%$ & & $0.2 \%$ & $0.8 \%$ & $264 \%$ \\
48 & 273 & 124 & $120 \%$ & & $14 \%$ & $19 \%$ & $39 \%$ \\
64 & 81 & 76 & $7 \%$ & & $17 \%$ & $26 \%$ & $56 \%$ \\
96 & 74 & 58 & $27 \%$ & & $46 \%$ & $49 \%$ & $8 \%$ \\
128 & 73 & 70 & $4 \%$ & & $52 \%$ & $50 \%$ & $-1.2 \%$ \\
256 & 106 & 115 & $-7 \%$ & & $57 \%$ & $55 \%$ & $-3 \%$ \\
\hline Best performance & $\mathbf{7 3}$ & $\mathbf{5 8}$ & $\mathbf{2 5 \%}$ & & $\mathbf{5 7 \%}$ & $\mathbf{5 5 \%}$ & $\mathbf{- 3 \%}$ \\
\hline
\end{tabular}

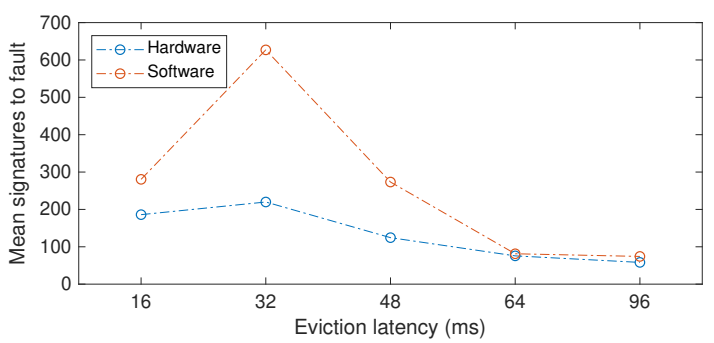

Figure 10: Mean number of signatures to fault at various eviction intervals.

a faulty signature is produced and the ultimate probability of success of an attack within 1000 signatures against a random key in a randomly selected chunk of memory within a row known to be vulnerable to Rowhammer. With an eviction interval of $96 \mathrm{~ms}$, the JackHammer attack achieves the lowest average number of signatures before a fault, at only $58,25 \%$ faster than the best performance of the CPU Rowhammer. The CPU attack is impeded significantly by shorter eviction latency, while the JackHammer implementation is not, indicating that on systems where the DRAM row refresh rate has been increased to protect against memory faults and Rowhammers, JackHammer likely offers substantially improved attack performance. Figure 10 highlights the mean number of signatures until a faulty signature for the $16 \mathrm{~ms}$ to $96 \mathrm{~ms}$ range of eviction latency.

\section{Cache Attacks on Intel FPGA-CPU Platforms}

In Section 3.3.1, we reverse-engineered the behavior of the memory subsystem on current Arria 10 based FPGA-CPU platforms. In this section, we systematically analyze cache attacks exploitable by an AFU- or CPU-based attacker attacking the CPU or FPGA, respectively, and demonstrate a cache covert channel from FPGA to CPU. At last, we discuss the viability of intra-FPGA cache attacks. Table 3 summarizes our findings.

To measure memory access latency on the FPGA, we designed a timer clocked at $200 \mathrm{MHz} / 400 \mathrm{MHz}$. The advantage of this hardware timer is that it runs uninterruptible in parallel to all other CPU or FPGA operations. Therefore, the timer precisely counts FPGA clock cycles, while timers on the CPU, such as rdtsc, may yield noisier measurements due to interruptions by the OS and the CPU's out-of-order pipeline.

\subsection{Cache Attacks from FPGA PAC to CPU}

The Intel PAC has access to one PCIe lane that connects it to the main memory of the system through the CPU's LLC. The CCI-P documentation [Int18a] mentions a timing 


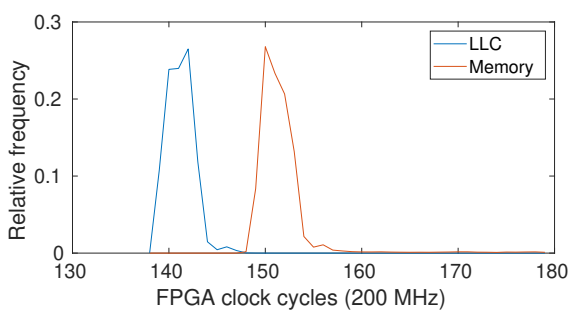

Figure 11: Latency for PCIe read requests on an FPGA PAC served by the CPU's LLC or main memory.

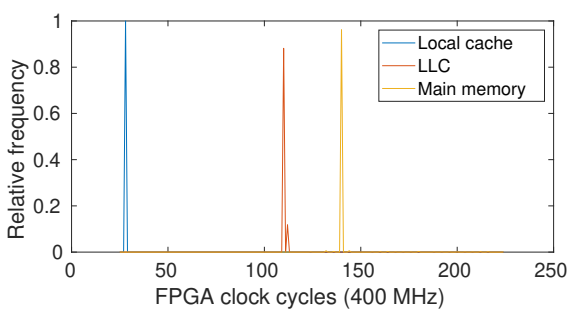

Figure 12: Latency for UPI read requests on an integrated Arria 10 served by the FPGA's local cache, CPU's LLC, or main memory.

difference for memory requests served by the CPU's LLC and those served by the main memory. Using our timer we verified the suggested differences as shown in Figure 11. Accesses to the LLC take between 139 and 145 cycles; accesses to main memory take 148 to 158 cycles. These distinct distributions of access latency form the basis of cache attacks, as they enable an attacker to tell which part of the memory subsystem served a particular memory request. Our results indicate that FPGA-based attackers can precisely distinguish memory responses served by the LLC from those served by main memory.

In addition to probing, some way of influencing the state of the cache is needed to perform cache attacks. We investigated all possibilities of cache interaction offered by the CCI-P interface on an FPGA PAC and found that cache lines read by the AFU from the main memory will not get cached. While this behavior is not usable for cache attacks, it boosts Rowhammer performance as we saw in Section 4. On the other hand, cache lines written by an AFU on the PAC end up in the LLC with nearly $100 \%$ probability. The reason for this behavior was already discussed together with the analysis of the caching hints. This can be used to evict other cache lines from the cache and perform eviction based attacks like Evict+Time, Evict+Reload, and Prime+Probe. For E+T, DMA writes can be used to evict a cache line and our hardware timer measures the victim's execution time. Even though an AFU cannot load data into the LLC, E+R can be performed as the purpose of reloading a cache line is to learn the latency and not literally reloading the cache line. So the primitives for $\mathrm{E}+\mathrm{R}$ on the FPGA are DMA writes and timing DMA reads with a hardware timer. $\mathrm{P}+\mathrm{P}$ can be performed using DMA writes and timing reads. In the case where DDIO limits the number of accessible ways per cache set, other DDIO-enabled peripherals are attackable. Flush-based attacks like Flush+Reload or Flush+Flush cannot be performed by an AFU as CCI-P does not offer a flush instruction.

\subsection{Cache Attacks from Integrated Arria 10 FPGA to CPU}

The integrated Arria 10 has access to two PCIe lanes (each functioning much like the PCIe lane on the FPGA PAC) and one UPI lane connecting it to the CPU's memory subsystem.

Table 3: Summary of our cache attacks analysis: OPAE accelerates eviction set construction by making huge pages and physical addresses available to userspace.

\begin{tabular}{cccc}
\hline Attacker & Target & Channel & Attack \\
\hline FPGA PAC AFU & CPU LLC & PCIe & E+T, E+R, P+P \\
Integrated FPGA AFU & CPU LLC & UPI & E+T, E+R, P+P \\
Integrated FPGA AFU & CPU LLC & PCIe & E+T, E+R, P+P \\
CPU & FPGA Cache & UPI & F+R, F+F \\
Integrated FPGA AFU & FPGA Cache & CCI-P & E+T, E+R, P+P \\
\hline
\end{tabular}




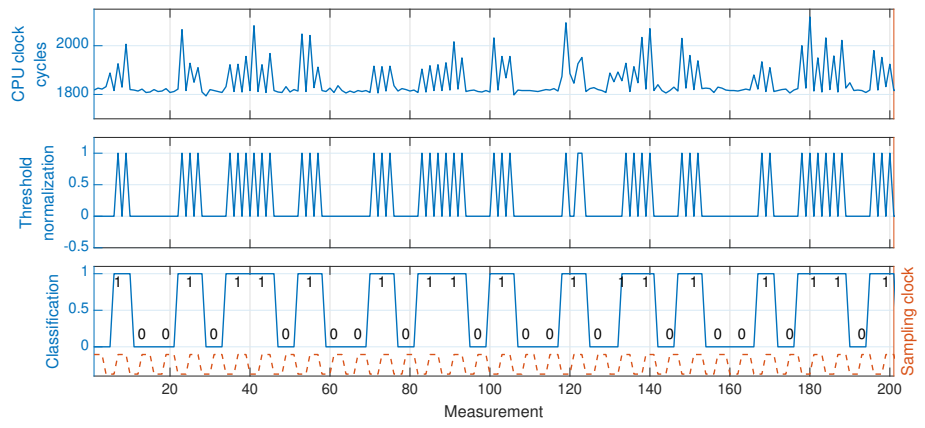

Figure 13: Covert channel measurements and decoding. The AFU sends each bit three times, which results in three peaks at the receiver if a ' 1 ' is transmitted (middle plot).

It also has its own additional cache on the FPGA accessible over UPI (cf. Section 3.3).

By timing memory requests from the AFU using our hardware timer, we show that distinct delays for the different levels of the memory subsystem exist. Both PCIe lanes have delays similar to those measured on a PAC (cf. Figure 11). Our memory access latency measurements for the UPI lane, depicted in Figure 12, show an additional peak for requests being answered by the FPGA's local cache. The two peaks for LLC and main memory accesses are likely narrower and further apart than in the PCIe case because UPI, Intel's proprietary high-speed processor interconnect, is an on-chip and inter-CPU bus only connecting CPUs and FPGAs. On all interfaces, read requests, again, are not usable for evicting cache lines from the LLC. DMA writes, however, can be used to alter the LLC on the CPU. Because the UPI and PCIe lanes behave much like the PCIe lane on a PAC, we state the same attack scenarios $(\mathrm{E}+\mathrm{T}, \mathrm{E}+\mathrm{R}, \mathrm{P}+\mathrm{P})$ to be viable on the integrated Arria 10.

\subsubsection{Constructing a Covert Channel from AFU to CPU}

The fact that an AFU can place data in at least one way per LLC slice allows us to construct a covert channel from the AFU to a co-operating process on the CPU using side effects of the LLC. To do so, we designed an AFU that writes a fixed string to a pre-configured cache line whenever a ' 1 ' is transmitted and stays quiet whenever a ' 0 ' is sent. Using this technique, the AFU sends messages which can be read by the CPU. For the rest of this section, we will refer to the address the AFU writes to as the target address.

The receiver process ${ }^{7}$ first constructs an eviction set for the set/slice-pair the target address is in. To find an eviction set, we run a slightly modified version of Algorithm 1 using Test 1 in [VKM19]. Using the OPAE API to allocate hugepages and get physical addresses (cf. Section 3.2) allows us to construct the eviction set from a rather small set of candidate addresses all belonging to the same set.

We construct the covert channel on the integrated platform as the LLC of the CPU is inclusive. Additionally, the receiver has access to the target address via shared memory to have the receiver test its eviction set against the target address directly. This way, we do not need to explicitly identify the target address's LLC slice. In a real-world scenario, either the slice selection function has to be known [HWH13, IES15b, IGG ${ }^{+} 15$ ] or eviction sets for all slices have to be constructed by seeking conflicting addresses [ $\left.\mathrm{LYG}^{+} 15, \mathrm{OKSK} 15\right]$. The time penalty introduced by monitoring all cache sets can be prevented by multi-threading.

Next, the receiver primes the LLC with the eviction set found and probes the set in an endless loop. Whenever the execution time of a probe is above a certain threshold, the receiver assumes that the eviction of one of its eviction set addresses was the result of

\footnotetext{
${ }^{7}$ This process is not the software process directly communicating with the AFU over OPAE/CCI-P.
} 
the AFU writing to the target address and therefore interprets this as receiving a ' 1 '. If the probe execution time stays below the threshold, a ' 0 ' is detected as no eviction of the eviction set addresses occurred. An example measurement of the receiver and its decoding steps are depicted in Figure 13.

To ease decoding and visualization of results, the AFU sends every bit thrice and the CPU uses six probes to detect all three repetitions. This high level of redundancy comes at the expense of speed, as we achieve a bandwidth of about $94.98 \mathrm{kBit} / \mathrm{s}$, which is low when compared to other work [WXW12, $\left.\mathrm{LYG}^{+} 15, \mathrm{MWS}^{+} 17\right]$. The throughput can be increased by reducing the three redundant writes per bit from the AFU as well as by increasing the transmission frequency further to reduce the redundant CPU probes per AFU write. Also, multiple cache sets can be used in parallel to encode several bits at once. The synchronization problem can be solved by using one cache set as the clock, where the AFU writes an alternating bit pattern [TVT19]. An average probe on the CPU takes 1855 clock cycles. With the CPU operating in the range of $2.8-3.4 \mathrm{GHz}$, this results in a theoretic throughput of $1.5-1.8 \mathrm{MBit} / \mathrm{s}$. On the other side, the AFU can on average send one write request every 10 clock cycles without filling the CCI-P PCIe buffer and thereby losing the write pattern. In theory, this makes the AFU capable of sending $40 \mathrm{MBit} / \mathrm{s}$ over the covert channel when clocked at $400 \mathrm{MHz} .^{8}$

Even though caching hints for memory writes are being ignored by the Blue Region, an $\mathrm{AFU}$ can place data in the LLC because the $\mathrm{CPU}$ is configured to handle write requests as if WrPush_I is set, allowing for producing evictions in the LLC. We corroborated our findings by establishing a covert channel between the AFU and the CPU with a bandwidth of $94.98 \mathrm{kBit} / \mathrm{s}$. By exposing physical addresses to the user and by enabling hugepages, OPAE further eases eviction set finding from userspace.

\subsection{Cache Attacks from CPU to Integrated Arria 10 FPGA}

We also investigated the CPU's capabilities to run cache attacks against the coherent cache on the integrated Arria 10 FPGA. First, we measured the memory access latency depending on the location of the address accessed using the rdtsc instruction. The results in Figure 14a show that the CPU can clearly distinguish where an accessed address is located. Therefore, the CPU is capable of probing a memory address that may or may not be present in the local FPGA cache. It is interesting to note that requests to main memory return faster than those going to the FPGA cache. This can be explained by the much slower clock speed of the FPGA running at $400 \mathrm{MHz}$ while the CPU operates at $1.2-3.4 \mathrm{GHz}$. Another explanation is that our test platform is one of the prototypes and the coherency protocol implementation of the Blue Region is still buggy. As nearly all known cache attack techniques rely on some form of probing phase, the capability to distinguish location of data is a good step in the direction of having a fully working cache attack from the CPU against the FPGA cache.

Besides the capability of probing the FPGA cache, we also need a way of flushing, priming, or evicting cache lines to put the FPGA cache into a known state. While the AFU can control which data is cached locally by using caching hints, there is no such option documented for the CPU. Therefore, priming the FPGA cache to evict cache lines is not possible. This disables all eviction-based cache attacks. However, as the CPU has a clfush instruction, we can use it to flush cache lines from the FPGA cache, because it is coherent with the LLC. Hence, we can flush and probe cache lines located in the FPGA cache. This enables us to run a Flush+Reload attack against the victim AFU where the addresses used by the AFU get flushed before the execution of the AFU. After the execution, the attacker then probes all previously flushed addresses to learn which

\footnotetext{
${ }^{8}$ This is a worst-case scenario where every transmitted bit is a '1'-bit. For a random message, this estimation goes up again as '0'-bits do not fill the buffer, allowing for faster transmission.
} 


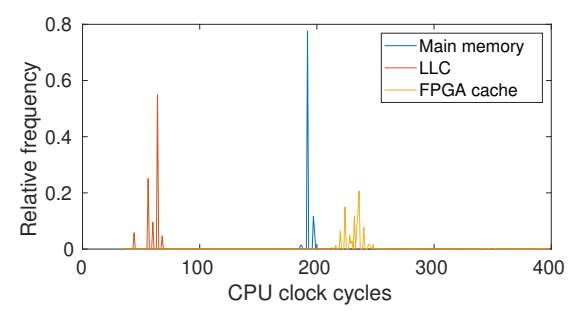

(a) Memory access latency on the CPU with data being present in FPGA local cache, CPU LLC, or main memory.

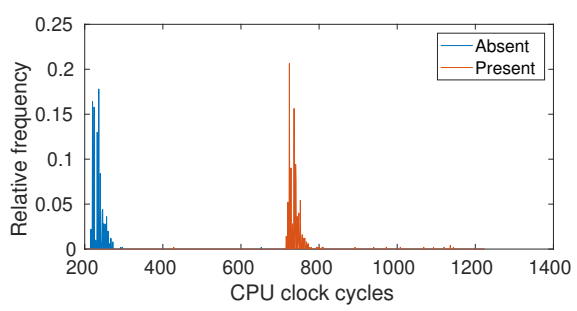

(b) The flush execution time on the CPU with the flushed address being absent or present in the FPGA cache.

Figure 14: Memory access and flush execution latency measured from a Broadwell CPU with integrated Arria 10.

addresses were used during the AFU execution. Another possible cache attack is the more efficient Flush+Flush attack. Additionally, we expect the attack to be more precise as flushing a cache line that is present in the FPGA cache takes about $500 \mathrm{CPU}$ clock cycles longer than flushing a cache line that is not (cf. Figure 14b), while the latency difference between memory and FPGA cache accesses adds up to only about 50-70 CPU clock cycles.

In general, the applicability of $\mathrm{F}+\mathrm{R}$ and $\mathrm{F}+\mathrm{F}$ is limited to shared memory scenarios. For example, two users on the same CPU might share an instantiation of a library that uses an $\mathrm{AFU}$ for acceleration of a process that should remain private, like training a machine learning model with confidential data or performing cryptographic operations.

\subsection{Intra-FPGA Cache Side-Channels}

As soon as FPGAs support simultaneous multi-tenancy, that is, the capability to place two AFUs from different users on the same FPGA at the same time, the possibility of intra-FPGA cache attacks arises. As the cache on the integrated Arria 10 is directly mapped and only $128 \mathrm{kB}$ in size, finding eviction sets becomes trivial when giving the attacker AFU access to huge pages. As this is the default behavior of the OPAE driver when allocating more than one memory page at once, we assume that it is straightforward to run eviction based attacks like Evict+Time or Prime+Probe against a neighboring AFU to e.g. extract information about a machine learning model. Flush-based attacks would still be impossible due to the lack of a flush instruction in CCI-P.

\section{Countermeasures}

Hardware Monitors Microarchitectural attacks against CPUs leave traces in hardware performance counters (HPCs) like cache hit and miss counters. Previous works have paired these HPCs with machine learning techniques to build real-time detectors for these attacks [BIME18, CSY16, ZZL16, GMES19]. In some cases, CPU HPCs may be able to trace incoming attacks from FPGAs. While HPCs do not exist in the same form on the Arria $10 \mathrm{GX}$ platforms, they could be implemented by the FIM. A system combining FPGA and CPU HPCs could provide thorough monitoring of the FPGA-CPU interface.

Increasing DRAM Row Refresh Rate An approach to reduce the impact of Rowhammer is increasing the DRAM refresh rate. DDR3 and DDR4 specifications require that each row is refreshed at least every $64 \mathrm{~ms}$, but many systems can be configured to refresh each row every 32 or $16 \mathrm{~ms}$ for better memory stability. When we measured the performance of our fault injection attack in Section 5 , we measured the performance with varying intervals between evictions of the targeted data, simulating equivalent intervals in row refresh rate, since each eviction causes a subsequent row refresh when the memory is read by the victim 
program. Table 2 shows that under $1 \%$ of attempted Rowhammers from both CPU and FPGA were successful with an eviction interval of $32 \mathrm{~ms}$, compared to $14 \%$ of CPU attacks and $26 \%$ of FPGA attacks with an interval of $64 \mathrm{~ms}$, suggesting that increasing the row refresh rate would significantly impede even the more powerful FPGA Rowhammer.

Cache Partitioning and Pinning Several cache partitioning mechanisms have been proposed to protect CPUs against cache attacks. While some are implementable in software [KPMR12, YWCL14, ZRZ16, KLA $\left.{ }^{+} 18\right]$ others require hardware support [LGY ${ }^{+} 16$, $\left.\mathrm{GRLZ}^{+} 17, \mathrm{GLS}^{+} 17\right]$. When trying to protect FPGA caches against cache attacks, hardware-based approaches should be taken into special consideration. For example, the FIM could partition the FPGA's cache into several security domains, such that each AFU can only use a subset of the cache lines in the local cache. Another approach would introduce an additional flag to the $\mathrm{CCl}-\mathrm{P}$ interface telling the local caching agent which cache lines to pin to the cache.

Disabling Hugepages and Virtualizing AFU Address Space Intel is aware of the fact that making physical addresses available to userspace through OPAE has negative security consequences [Int17]. Additionally to exposing physical addresses, OPAE makes heavy use of hugepages to ensure physical address continuity of buffers shared with the AFU. However, it is well known that disabling hugepages increases the barrier of finding eviction sets [IES15a, $\left.\mathrm{LYG}^{+} 15\right]$ which in turn makes cache attacks and Rowhammer more difficult. We suggest disabling OPAE's usage of hugepages. To do so, the AFU address space has to be virtualized independent of the presence of virtual environments.

Protection Against Bellcore Attack Defenses against fault injection attacks proposed in the original Bellcore whitepaper [BDL97] include verifying the signature before releasing it, and random padding of the message before signing, which ensures that no unique message is ever signed twice and that the exact plaintext cannot be easily determined. OpenSSL protects against the Bellcore attack by verifying the signature with its plaintext and public key and recomputing the exponentiation by a slower but safer single exponentiation instead of by the CRT if verification does not match [CDFG18]. After we reported the vulnerability to WolfSSL, they issued a patch in version 4.3.0 including a signature verification to protect against Bellcore-style attacks.

\section{Conclusion}

In this work, we show that modern FPGA-CPU hybrid systems can be more vulnerable to well-known hardware attacks that are traditionally seen on CPU-only systems. We show that the shared cache systems of the Arria $10 \mathrm{GX}$ and its host CPU present possible CPU to FPGA, FPGA to CPU, and FPGA to FPGA attack vectors. For Rowhammer, we show that the Arria $10 \mathrm{GX}$ is capable of causing more DRAM faults in less time than modern CPUs. Our research indicates that defense against hardware side-channels is just as essential for modern FPGA systems as it is for modern CPUs. Of course, the security of any device physically installed in a system, like a network card or graphics card, is important, but FPGAs present additional security challenges due to their inherently flexible nature. From a security perspective, a user-configurable FPGA on a cloud system needs to be treated with at least as much care and caution as a user-controlled CPU thread, as it can exploit many of the same vulnerabilities.

\section{Acknowledgments}

This research received partial funding, hardware donations, and extremely useful advice from Intel and its employees. We would like to especially thank Alpa Trivedi from Intel. 
Daniel Moghimi was supported by the National Science Foundation under grant no. CNS1814406. This work was partially supported by the German Research Foundation (DFG) project number 427774779 .

\section{References}

$\left[\mathrm{ABF}^{+}\right.$03] Christian Aumüller, Peter Bier, Wieland Fischer, Peter Hofreiter, and JeanPierre Seifert. Fault attacks on RSA with CRT: Concrete results and practical countermeasures. In Burton S. Kaliski, Çetin K. Koç, and Christof Paar, editors, Cryptographic Hardware and Embedded Systems - CHES 2002, pages 260-275, Berlin, Heidelberg, 2003. Springer Berlin Heidelberg.

[Ali19] Alibaba Cloud. FPGA-based compute-optimized instance families. https: //www.alibabacloud.com/help/doc-detail/108504.html, 2019. Access: 2019-10-15.

[Ama17] Amazon Web Services. Amazon EC2 F1 instances. https://aws.amazon. com/ec2/instance-types/f1/, 2017. Access: 2019-10-12.

[BDL97] Dan Boneh, Richard A. DeMillo, and Richard J. Lipton. On the Importance of Checking Cryptographic Protocols for Faults. In Walter Fumy, editor, Advances in Cryptology - EUROCRYPT' '97, pages 37-51, Berlin, Heidelberg, 1997. Springer Berlin Heidelberg.

[BH09] Billy Bob Brumley and Risto M. Hakala. Cache-Timing Template Attacks. In Mitsuru Matsui, editor, Advances in Cryptology - ASIACRYPT 2009, pages 667-684, Berlin, Heidelberg, 2009. Springer Berlin Heidelberg.

[BIME18] Samira Briongos, Gorka Irazoqui, Pedro Malagón, and Thomas Eisenbarth. CacheShield: Detecting Cache Attacks through Self-Observation. In Proceedings of the Eighth ACM Conference on Data and Application Security and Privacy, CODASPY '18, pages 224-235, New York, NY, USA, 2018. Association for Computing Machinery.

[BM16] Sarani Bhattacharya and Debdeep Mukhopadhyay. Curious Case of Rowhammer: Flipping Secret Exponent Bits Using Timing Analysis. In Benedikt Gierlichs and Axel Y. Poschmann, editors, Cryptographic Hardware and Embedded Systems - CHES 2016, pages 602-624, Berlin, Heidelberg, 2016. Springer Berlin Heidelberg.

[BM18] Sarani Bhattacharya and Debdeep Mukhopadhyay. Advanced Fault Attacks in Software: Exploiting the Rowhammer Bug. In Sikhar Patranabis and Debdeep Mukhopadhyay, editors, Fault Tolerant Architectures for Cryptography and Hardware Security, pages 111-135. Springer Singapore, Singapore, 2018.

[BvdPSY14] Naomi Benger, Joop van de Pol, Nigel P. Smart, and Yuval Yarom. "Ooh Aah... Just a Little Bit" : A Small Amount of Side Channel Can Go a Long Way. In Lejla Batina and Matthew Robshaw, editors, Cryptographic Hardware and Embedded Systems - CHES 2014, pages 75-92, Berlin, Heidelberg, 2014. Springer Berlin Heidelberg.

[CDFG18] Sébastien Carré, Matthieu Desjardins, Adrien Facon, and Sylvain Guilley. OpenSSL Bellcore's Protection Helps Fault Attack. In 2018 21st Euromicro Conference on Digital System Design (DSD), pages 500-507, August 2018. 
[CSY16] Marco Chiappetta, Erkay Savas, and Cemal Yilmaz. Real time detection of cache-based side-channel attacks using hardware performance counters. Applied Soft Computing, 49:1162 - 1174, 2016.

[FGBR18] Pietro Frigo, Cristiano Giuffrida, Herbert Bos, and Kaveh Razavi. Grand Pwning Unit: Accelerating Microarchitectural Attacks with the GPU. In 2018 IEEE Symposium on Security and Privacy (SP), pages 195-210, May 2018 .

[GER19] Ilias Giechaskiel, Ken Eguro, and Kasper B. Rasmussen. Leakier Wires: Exploiting FPGA Long Wires for Covert- and Side-Channel Attacks. ACM Trans. Reconfigurable Technol. Syst., 12(3), August 2019.

[GES17] Berk Gülmezoğlu, Thomas Eisenbarth, and Berk Sunar. Cache-Based Application Detection in the Cloud Using Machine Learning. In Proceedings of the 2017 ACM on Asia Conference on Computer and Communications Security, ASIA CCS '17, pages 288-300, New York, NY, USA, 2017. Association for Computing Machinery.

[GLS $\left.{ }^{+} 17\right]$ Daniel Gruss, Julian Lettner, Felix Schuster, Olya Ohrimenko, Istvan Haller, and Manuel Costa. Strong and Efficient Cache Side-Channel Protection using Hardware Transactional Memory. In 26th USENIX Security Symposium (USENIX Security 17), pages 217-233, Vancouver, BC, August 2017. USENIX Association.

[GLS $\left.{ }^{+} 18\right]$ Daniel Gruss, Moritz Lipp, Michael Schwarz, Daniel Genkin, Jonas Juffinger, Sioli O'Connell, Wolfgang Schoechl, and Yuval Yarom. Another Flip in the Wall of Rowhammer Defenses. In 2018 IEEE Symposium on Security and Privacy (SP), pages 245-261, May 2018.

[GMES19] Berk Gülmezoğlu, Ahmad Moghimi, Thomas Eisenbarth, and Berk Sunar. FortuneTeller: Predicting Microarchitectural Attacks via Unsupervised Deep Learning, 2019.

[GMM16] Daniel Gruss, Clémentine Maurice, and Stefan Mangard. Rowhammer.js: A Remote Software-Induced Fault Attack in JavaScript. In Juan Caballero, Urko Zurutuza, and Ricardo J. Rodríguez, editors, Detection of Intrusions and Malware, and Vulnerability Assessment, pages 300-321, Cham, 2016. Springer International Publishing.

[GMWM16] Daniel Gruss, Clémentine Maurice, Klaus Wagner, and Stefan Mangard. Flush+Flush: A Fast and Stealthy Cache Attack. In Juan Caballero, Urko Zurutuza, and Ricardo J. Rodríguez, editors, Detection of Intrusions and Malware, and Vulnerability Assessment, pages 279-299, Cham, 2016. Springer International Publishing.

[GOT17] Dennis R. E. Gnad, Fabian Oboril, and Mehdi B. Tahoori. Voltage drop-based fault attacks on FPGAs using valid bitstreams. In 2017 27th International Conference on Field Programmable Logic and Applications (FPL), pages 1-7, September 2017.

[GRLZ+17] Marc Green, Leandro Rodrigues-Lima, Andreas Zankl, Gorka Irazoqui, Johann Heyszl, and Thomas Eisenbarth. AutoLock: Why Cache Attacks on ARM Are Harder Than You Think. In 26th USENIX Security Symposium (USENIX Security 17), pages 1075-1091, Vancouver, BC, August 2017. USENIX Association. 
[GZES17] Berk Gülmezoğlu, Andreas Zankl, Thomas Eisenbarth, and Berk Sunar. PerfWeb: How to Violate Web Privacy with Hardware Performance Events. In Simon N. Foley, Dieter Gollmann, and Einar Snekkenes, editors, Computer Security - ESORICS 2017, pages 80-97, Cham, 2017. Springer International Publishing.

[Huf18] Jennifer Huffstetler. Intel processors and FPGAs - better together. https://itpeernetwork.intel.com/intel-processors-fpgabetter-together/, 2018. accessed: 21-05-19.

[HWH13] Ralf Hund, Carsten Willems, and Thorsten Holz. Practical Timing Side Channel Attacks against Kernel Space ASLR. In 2013 IEEE Symposium on Security and Privacy (SP), pages 191-205, May 2013.

[IES15a] Gorka Irazoqui, Thomas Eisenbarth, and Berk Sunar. S\$A: A Shared Cache Attack That Works across Cores and Defies VM Sandboxing - and Its Application to AES. In 2015 IEEE Symposium on Security and Privacy (SP), pages 591-604, May 2015.

[IES15b] Gorka Irazoqui, Thomas Eisenbarth, and Berk Sunar. Systematic Reverse Engineering of Cache Slice Selection in Intel Processors. In 2015 Euromicro Conference on Digital System Design (DSD), pages 629-636, August 2015.

[IES16] Gorka Irazoqui, Thomas Eisenbarth, and Berk Sunar. Cross Processor Cache Attacks. In Proceedings of the 11th ACM on Asia Conference on Computer and Communications Security, ASIA CCS '16, pages 353-364, New York, NY, USA, 2016. Association for Computing Machinery.

$\left[\right.$ İGI $\left.^{+} 15\right]$ Mehmet Sinan İnci, Berk Gülmezoğlu, Gorka Irazoqui, Thomas Eisenbarth, and Berk Sunar. Seriously, get off my cloud! Cross-VM RSA Key Recovery in a Public Cloud. IACR Cryptology ePrint Archive, 2015.

[İGI ${ }^{+}$16] Mehmet Sinan İnci, Berk Gülmezoğlu, Gorka Irazoqui, Thomas Eisenbarth, and Berk Sunar. Cache Attacks Enable Bulk Key Recovery on the Cloud. In Benedikt Gierlichs and Axel Y. Poschmann, editors, Cryptographic Hardware and Embedded Systems - CHES 2016, pages 368-388, Berlin, Heidelberg, 2016. Springer Berlin Heidelberg.

[Int17] Intel. Open Programmable Acceleration Engine, 1.1.2 edition, 2017. https: //opae.github.io/1.1.2/index.html.

[Int18a] Intel. Acceleration Stack for Intel Xeon CPU with FPGAs Core Cache Interface (CCI-P) Reference Manual, 1.2 edition, December 2018. https://www.intel.com/content/dam/www/programmable/us/en/ pdfs/literature/manual/mnl-ias-ccip.pdf.

[Int18b] Intel. Accelerator cards that fit your performance needs. https://www. intel. com/content/www/us/en/programmable/solutions/accelerationhub/platforms.html, 2018. accessed: 21-05-19.

[JC-17] JC-42.6 Low Power Memories Comittee. Low Power Double Data Rate 4 (LPDDR4). Standard JESD209-4B, JEDEC Solid State Technology Association, March 2017.

[KGA $\left.{ }^{+} 19\right]$ Michael Kurth, Ben Gras, Dennis Andriesse, Cristiano Giuffrida, Herbert Bos, and Kaveh Razavi. NetCAT: Practical Cache Attacks from the Network. https://download.vusec.net/papers/netcat_sp20.pdf, 2019. 
[KGT18] Jonas Krautter, Dennis R. E. Gnad, and Mehdi B. Tahoori. FPGAhammer: Remote Voltage Fault Attacks on Shared FPGAs, suitable for DFA on AES. IACR Transactions on Cryptographic Hardware and Embedded Systems, 2018(3):44-68, August 2018.

[KJJ99] Paul Kocher, Joshua Jaffe, and Benjamin Jun. Differential Power Analysis. In Michael Wiener, editor, Advances in Cryptology - CRYPTO' 99, pages 388-397, Berlin, Heidelberg, 1999. Springer Berlin Heidelberg.

$\left[\mathrm{KLA}^{+} 18\right]$ Vladimir Kiriansky, Ilia Lebedev, Saman Amarasinghe, Srinivas Devadas, and Joel Emer. DAWG: A Defense Against Cache Timing Attacks in Speculative Execution Processors. In 2018 51st Annual IEEE/ACM International Symposium on Microarchitecture (MICRO), pages 974-987, October 2018.

[KPMR12] Taesoo Kim, Marcus Peinado, and Gloria Mainar-Ruiz. STEALTHMEM: System-Level Protection Against Cache-Based Side Channel Attacks in the Cloud. In 21st USENIX Security Symposium (USENIX Security 12), pages 189-204, Bellevue, WA, 2012. USENIX.

[LGS $\left.{ }^{+} 16\right] \quad$ Moritz Lipp, Daniel Gruss, Raphael Spreitzer, Clémentine Maurice, and Stefan Mangard. ARMageddon: Cache Attacks on Mobile Devices. In 25th USENIX Security Symposium (USENIX Security 16), pages 549-564, Austin, TX, August 2016. USENIX Association.

[LGY ${ }^{+}$16] Fangfei Liu, Qian Ge, Yuval Yarom, Frank Mckeen, Carlos Rozas, Gernot Heiser, and Ruby B. Lee. CATalyst: Defeating Last-Level Cache Side Channel Attacks in Cloud Computing. In 2016 IEEE International Symposium on High Performance Computer Architecture (HPCA), pages 406-418, March 2016.

[LYG ${ }^{+}$15] Fangfei Liu, Yuval Yarom, Qian Ge, Gernot Heiser, and Ruby B. Lee. LastLevel Cache Side-Channel Attacks are Practical. In 2015 IEEE Symposium on Security and Privacy (SP), pages 605-622, May 2015.

[MIE17] Ahmad Moghimi, Gorka Irazoqui, and Thomas Eisenbarth. CacheZoom: How SGX Amplifies the Power of Cache Attacks. In Wieland Fischer and Naofumi Homma, editors, Cryptographic Hardware and Embedded Systems CHES 2017, pages 69-90, Cham, 2017. Springer International Publishing.

[mit19] CVE-2019-19962. Available from MITRE, CVE-ID CVE-2019-19962., December 2019.

[Mul17] David Mulnix. Intel Xeon processor scalable family technical overview. https://software.intel.com/en-us/articles/intel-xeonprocessor-scalable-family-technical-overview, 2017. Accessed: 201907-10.

[MWS ${ }^{+}$17] Clémentine Maurice, Manuel Weber, Michael Schwarz, Lukas Giner, Daniel Gruss, Carlo Alberto Boano, Stefan Mangard, and Kay Romer. Hello from the Other Side: SSH over Robust Cache Covert Channels in the Cloud. In 24th Annual Network and Distributed System Security Symposium (NDSS), San Diego, CA, 2017. Internet Society.

[OKSK15] Yossef Oren, Vasileios P. Kemerlis, Simha Sethumadhavan, and Angelos D. Keromytis. The Spy in the Sandbox: Practical Cache Attacks in JavaScript and Their Implications. In Proceedings of the 22nd ACM SIGSAC Conference on Computer and Communications Security, CCS '15, pages 1406-1418, New York, NY, USA, 2015. Association for Computing Machinery. 
[OST06] Dag Arne Osvik, Adi Shamir, and Eran Tromer. Cache Attacks and Countermeasures: The Case of AES. In David Pointcheval, editor, Topics in Cryptology - CT-RSA 2006, pages 1-20, Berlin, Heidelberg, 2006. Springer Berlin Heidelberg.

[PGM $\left.{ }^{+} 16\right]$ Peter Pessl, Daniel Gruss, Clémentine Maurice, Michael Schwarz, and Stefan Mangard. DRAMA: Exploiting DRAM Addressing for Cross-CPU Attacks. In 25th USENIX Security Symposium (USENIX Security 16), pages 565-581, Austin, TX, August 2016. USENIX Association.

$\left[\mathrm{PRP}^{+}\right.$19] George Provelengios, Chethan Ramesh, Shivukumar B. Patil, Ken Eguro, Russell Tessier, and Daniel Holcomb. Characterization of Long Wire Data Leakage in Deep Submicron FPGAs. In Proceedings of the 2019 ACM/SIGDA International Symposium on Field-Programmable Gate Arrays, FPGA '19, pages 292-297, New York, NY, USA, 2019. Association for Computing Machinery.

$\left[\mathrm{RPD}^{+} 18\right]$ Chethan Ramesh, Shivukumar B. Patil, Siva Nishok Dhanuskodi, George Provelengios, Sébastien Pillement, Daniel Holcomb, and Russell Tessier. FPGA Side Channel Attacks without Physical Access. In 2018 IEEE 26th Annual International Symposium on Field-Programmable Custom Computing Machines (FCCM), pages 45-52, April 2018.

[RTSS09] Thomas Ristenpart, Eran Tromer, Hovav Shacham, and Stefan Savage. Hey, You, Get off of My Cloud: Exploring Information Leakage in Third-Party Compute Clouds. In Proceedings of the 16th ACM Conference on Computer and Communications Security, CCS '09, pages 199-212, New York, NY, USA, 2009. Association for Computing Machinery.

[Sch19] Michael Schwarz. PTEditor: A small library to modify all page-table levels of all processes from user space for x86_64 and ARMv8. https://github. com/misc0110/PTEditor, April 2019.

[SD15] Mark Seaborn and Thomas Dullien. Exploiting the DRAM rowhammer bug to gain kernel privileges. Black Hat, 15, 2015.

[SGMT18a] Falk Schellenberg, Dennis R. E. Gnad, Amir Moradi, and Mehdi B. Tahoori. An Inside Job: Remote Power Analysis Attacks on FPGAs. In 2018 Design, Automation Test in Europe Conference Exhibition (DATE), pages 1111-1116, March 2018.

[SGMT18b] Falk Schellenberg, Dennis R. E. Gnad, Amir Moradi, and Mehdi B. Tahoori. Remote Inter-Chip Power Analysis Side-Channel Attacks at Board-Level. In 2018 IEEE/ACM International Conference on Computer-Aided Design (ICCAD), pages 1-7, November 2018.

$\left[\mathrm{TSS}^{+}\right.$03] Yukiyasu Tsunoo, Teruo Saito, Tomoyasu Suzaki, Maki Shigeri, and Hiroshi Miyauchi. Cryptanalysis of DES Implemented on Computers with Cache. In Colin D. Walter, Çetin K. Koç, and Christof Paar, editors, Cryptographic Hardware and Embedded Systems - CHES 2003, pages 62-76, Berlin, Heidelberg, 2003. Springer Berlin Heidelberg.

[TVT19] Mohammadkazem Taram, Ashish Venkat, and Dean Tullsen. Packet Chasing: Spying on Network Packets over a Cache Side-Channel. arXiv preprint arXiv:1909.04841, 2019. 
[vdVFL $\left.{ }^{+} 16\right]$ Victor van der Veen, Yanick Fratantonio, Martina Lindorfer, Daniel Gruss, Clémentine Maurice, Giovanni Vigna, Herbert Bos, Kaveh Razavi, and Cristiano Giuffrida. Drammer: Deterministic Rowhammer Attacks on Mobile Platforms. In Proceedings of the 2016 ACM SIGSAC Conference on Computer and Communications Security, CCS '16, pages 1675-1689, New York, NY, USA, 2016. Association for Computing Machinery.

[VKM19] Pepe Vila, Boris Köpf, and José Francisco Morales. Theory and Practice of Finding Eviction Sets. In 2019 IEEE Symposium on Security and Privacy $(S P)$, pages 39-54, May 2019.

[WvWM11] Marc F. Witteman, Jasper G. J. van Woudenberg, and Federico Menarini. Defeating RSA Multiply-Always and Message Blinding Countermeasures. In Aggelos Kiayias, editor, Topics in Cryptology - CT-RSA 2011, pages 77-88, Berlin, Heidelberg, 2011. Springer Berlin Heidelberg.

[WXW12] Zhenyu Wu, Zhang Xu, and Haining Wang. Whispers in the Hyper-space: High-speed Covert Channel Attacks in the Cloud. In 21st USENIX Security Symposium (USENIX Security 12), pages 159-173, Bellevue, WA, 2012. USENIX.

[Xil19] Xilinx. Accelerator Cards. https://www.xilinx.com/products/boardsand-kits/accelerator-cards.html, 2019. Accessed: 2019-10-15.

[YF14] Yuval Yarom and Katrina Falkner. FLUSH+RELOAD: A High Resolution, Low Noise, L3 Cache Side-Channel Attack. In 23rd USENIX Security Symposium (USENIX Security 14), pages 719-732, San Diego, CA, August 2014. USENIX Association.

[YSG $\left.{ }^{+} 19\right]$ Mengjia Yan, Read Sprabery, Bhargava Gopireddy, Christopher Fletcher, Roy Campbell, and Josep Torrellas. Attack Directories, Not Caches: Side Channel Attacks in a Non-Inclusive World. In 2019 IEEE Symposium on Security and Privacy (SP), pages 888-904, May 2019.

[YWCL14] Ying Ye, Richard West, Zhuoqun Cheng, and Ye Li. COLORIS: A Dynamic Cache Partitioning System Using Page Coloring. In 2014 23rd International Conference on Parallel Architecture and Compilation Techniques (PACT), pages 381-392, August 2014.

[ZJRR12] Yinqian Zhang, Ari Juels, Michael K. Reiter, and Thomas Ristenpart. CrossVM Side Channels and Their Use to Extract Private Keys. In Proceedings of the 2012 ACM Conference on Computer and Communications Security, CCS '12, pages 305-316, New York, NY, USA, 2012. Association for Computing Machinery.

[ZRZ16] Ziqiao Zhou, Michael K. Reiter, and Yinqian Zhang. A Software Approach to Defeating Side Channels in Last-Level Caches. In Proceedings of the 2016 ACM SIGSAC Conference on Computer and Communications Security, CCS '16, pages 871-882, New York, NY, USA, 2016. Association for Computing Machinery.

[ZS18] Mark Zhao and G. Edward Suh. FPGA-Based Remote Power Side-Channel Attacks. In 2018 IEEE Symposium on Security and Privacy (SP), pages 229-244, May 2018. 
[ZZL16] Tianwei Zhang, Yinqian Zhang, and Ruby B. Lee. CloudRadar: A RealTime Side-Channel Attack Detection System in Clouds. In Fabian Monrose, Marc Dacier, Gregory Blanc, and Joaquin Garcia-Alfaro, editors, Research in Attacks, Intrusions, and Defenses (RAID), pages 118-140, Cham, 2016. Springer International Publishing. 